\title{
Influence of shell structure on the equation of state of matter at high pressures*
}

\author{
E. BoschI (**)
}

Received on February 21st, 1972

Summary. - We develop an equation of state of matter in the pressure range of geophysical interest introducing shell structure in the ThomasFermi-Dirac atom model.

Riassunto. - Viene sviluppata un'equazione di stato della nateria nell'intervallo di pressioni d'interesse geofisico, introducendo la struttura a strati nel modello atomico di Thomas-Fermi-Dirac.

The Thomas-Fermi model, in its original formulation, cannot give account for the existence of discrete energy levels for bound electrons or, in other words, is not sensitive to the effects of shell structure (2). It is also obvious that these effects are the principal cause of the inapplicability of the Thomas-Fermi-Dirac model in the pressure range of geophysical interest $\left(^{4}\right)$. Up today, these effects have been considered only from a qualitative point of view and the influence of them on the equation of state has not been determined. We believe that it is possible to solve consistently this problem by means of an appropriate modification of the normalization of the Thomas-Fermi-Dirac electronic charged cloud. As it is well-known, in the Thomas-FermiDirac theory, the number of the possible states per volume unit

(*) Paper presented at the "First European Earth and Planetary Physics Colloquium", Reading (U.K.), 30 March-2 April 1971.

(**) Istituto di Fisica, Università degli Studi di Bologna (Italy). 
of the electrons, whose momentum is between $p$ and $p+d p$, is $8 \pi p^{2} d p / h^{3}$. Their density is given by the Fermi-Dirac statistics:

$$
d \varrho=\frac{8 \pi p^{2} d p}{h^{s} \exp \left(\frac{E_{k i n}-e \varphi}{k T}+\mu\right)+1}
$$

where $E_{k i n}$ is the kinetic energy, $\varphi$ the Thomas-Fermi potential, $k$ the Boltzman constant, $T$ the temperature, and $\mu$ the chemical potential. If we consider an atom of atomic number $Z$, the integral of $d o$ over the entire phase-space atomic volume must satisfy the condition:

$$
\frac{32 \pi^{2}}{h^{3}} \int_{0}^{R} \int_{0}^{\infty} \frac{r^{2} d r p^{2} d p}{\exp \left(\frac{E_{k ! n}-e \varphi}{k T}+\mu\right)+1}=Z
$$

Furthermore the Poisson's equation gives another relation between the electron density and the potential.

Now we assume that only a part of the electrons in the atom can satisfy to the Thomas-Fermi-Dirac requirements. The assumption means that there is, for a given value of the pressure and of the temperature, some "critical radius", $R_{c}$, which divides the atom volume into two parts: an outer part containing electrons which can be actually considered as a charge cloud and an inner part containing electrons whose behaviour needs a quantum mechanical description. So, for given external conditions, we have $B$ "bound" electrons and $F$ "free" electrons, where $B$ and $F$ are linked by the obvious relation: $F+B=Z$. In more precise words we introduce a cutoff in the momentum phase space of the atom that means to change the lower limit of momentum integration, 0 , which appears in the normalization equation, to some value $p$.

In this manner we obtain the actual number of electrons, the so-called free electron, which can be treated statistically. Since the free electrons are contained in a continuum of energy levels, the use of the Thomas-Fermi-Dirac theory appears well justified (3).

The way to determine the counting of bound electrons is to use a trial potential $V$ in Schrodinger's equation, to obtain the energy 
levels corresponding to $V$, and calculate the number of bound electrons from the relations:

$$
N_{n l}=\frac{2(2 l+1)}{\exp \left(-E_{n l} / k T+\mu\right)+1}
$$

and

$$
B=\Sigma_{n l} N_{n l}
$$

where $n$ and $l$ are the principal and orbital quantum numbers, $N_{n l}$ is the average number of electrons in the subshell $(n l)$ and $B$ is the total number of bound electrons.

After these considerations, the Thomas-Fermi-Dirac normalization condition becomes:

$$
\begin{gathered}
\frac{32 \pi^{-}}{n^{3}} \int_{\int_{R_{c}}^{R}}^{\int_{R^{2}}^{\infty}} \frac{r^{2} d r p^{2} d p}{\exp \left(\frac{E_{k i n}-e V}{k T}+\mu\right)+1}+ \\
+\sum_{n l} \frac{2(2 l+1)}{\exp \left(-E_{n l} / k T+\mu\right)+1}=Z
\end{gathered}
$$

Searching the appropriate potential $V$ which appears in the last equation, it seems natural to take into consideration the ionization phenomena and to represent the passage from bound electrons to free electrons as some kind of ionization induced by pressure. This makes possible the task of finding an appropriate potential function (1):

$$
V=\left\{\begin{array}{l}
\frac{F e}{R}\left(\frac{R}{r}+\frac{R^{2}}{2 r^{2}}-\frac{3}{2}\right), \quad R_{c} \leqslant r \leqslant R \\
\frac{Z e}{r(1+A r)^{2}}+\frac{F e}{R}\left(\frac{r^{2}}{2 R^{2}}-A_{1}\right) ; \quad 0 \leqslant r \leqslant R_{c}
\end{array}\right.
$$

where $A_{1}$ is chosen to make $V$ continuous at $r-R_{c}$ :

$$
\dot{A}_{1}=\frac{Z R}{R_{c}\left(1+A R_{c}\right)^{2} W}-\frac{R}{R_{c}} \div \frac{3}{2} .
$$

By means of Poisson's equation:

$$
F=\frac{2 Z A R_{c}}{\left(1+A R_{c}\right)^{3}}+\frac{Z}{\left(1+A R_{c}\right)^{2}}
$$


where $A$ is a constant for a given value of $Z$. $R_{c}$ divides the atomic volume into two regions: an inner region around the nucleus where the electron density is high and due to bound electrons, and an outer region where the electron density is low and due to free electrons.

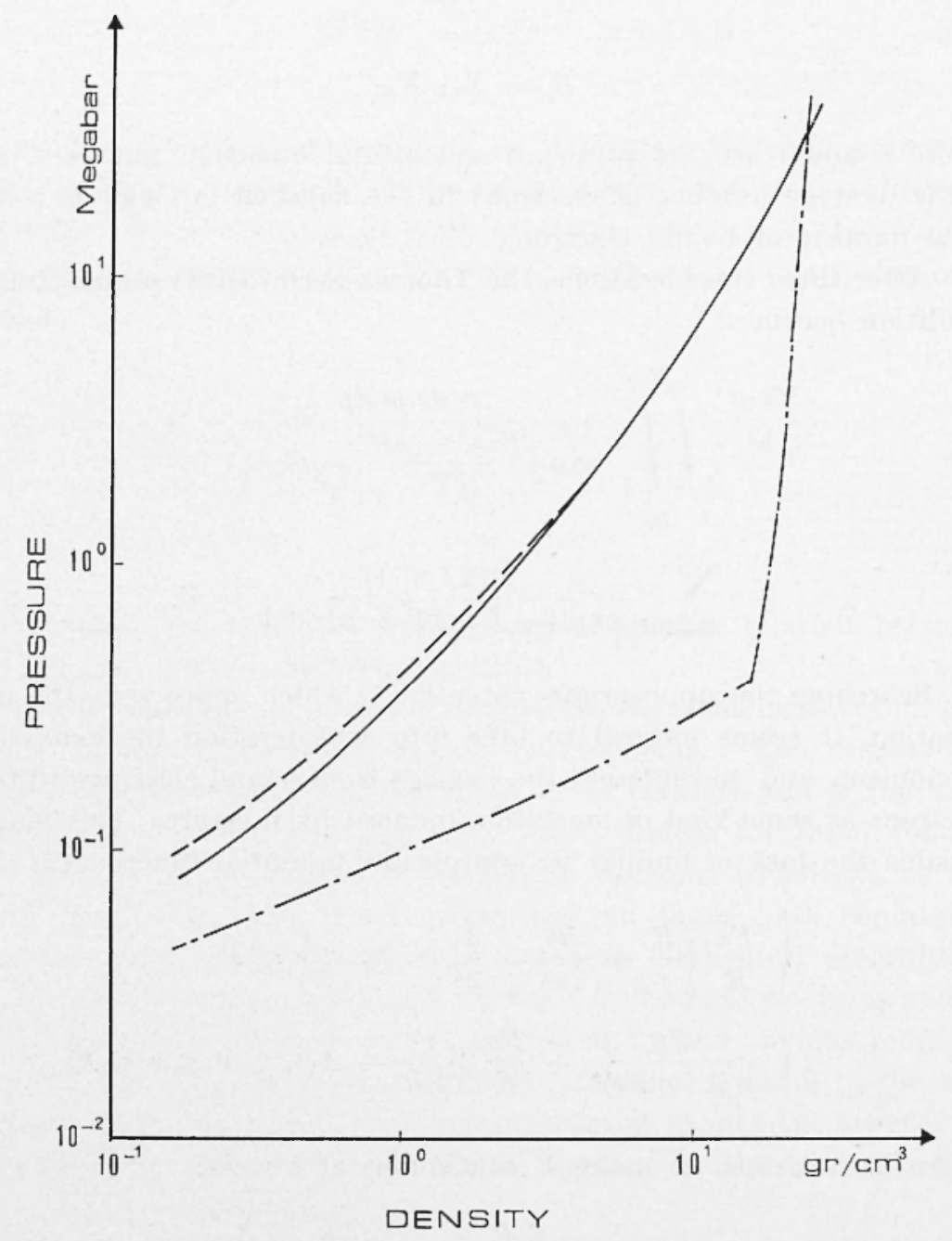

To perform the calculations, we use an iterative process starting from a trial value of the electron density. The figure shows the results obtained for an atom of atomic number 26 and puts in evidence the discrepancy between the Thomas-Fermi-Dirac model, represented by 
a continuous curve line, and the results of these considerations, represented by a broken and dotted line. The dotted line, near to the continuous one, represents a Thomas-Fermi-Dirac calculation with the potential $V$ made to check the internal consistency of the work.

\section{ACKNowledgeneNTs}

I wish to thank Prof. MI. Caputo for valuable discussions on this work.

\section{REFERENCES}

(1) Armstrong B. H., Jolnston R. R., Kelly P. S., 1967. - Progress in high temperature physics and chemistry, edited by Carl. A. Rouse, Pergamon Press.

(2) Boschi E., Caputo M., 1969. - "Nuovo Cimento", 1, 441.

(3) Boscin E., Caputo M., 1971. - Mantle and Core in Planetary Physics, edited by J. Coulomb and M. Caputo, Academic Press, Milano.

(4) Takeuciti II., Kayamori H., 1966. - "Journ. Geophys. Res. ", 71, 3985. 